Revue européenne des sciences sociales

European Journal of Social Sciences

XLIV-134 | 2006

Quel(s) défi(s) pour les sciences sociales à l'heure de la mondialisation?

\title{
Sélection, sélection des risques et assurance maladie
}

Peter Zweifel

\section{CpenEdition}

Journals

Édition électronique

URL : http://journals.openedition.org/ress/319

DOI : $10.4000 /$ ress.319

ISSN : 1663-4446

Éditeur

Librairie Droz

Édition imprimée

Date de publication : 1 juin 2006

Pagination : 317-334

ISBN : 9-782-600-01095-5

ISSN : 0048-8046

Référence électronique

Peter Zweifel, «Sélection, sélection des risques et assurance maladie », Revue européenne des sciences sociales [En ligne], XLIV-134 | 2006, mis en ligne le 15 octobre 2009, consulté le 19 avril 2019. URL : http://journals.openedition.org/ress/319 ; DOI : 10.4000/ress.319 
Peter ZWEIFEL

\section{SÉLECTION, SÉLECTION DES RISQUES ET ASSURANCE MALADIE ${ }^{1}$}

\section{INTRODUCTION}

L'assurance maladie sociale mise en place en Suisse est une grande expérience parce qu'elle cherche à instaurer la concurrence entre les assureurs dans un domaine où la plupart des pays ne l'admettent pas. Ceux qui misent sur la concurrence pour améliorer la performance de leurs systèmes de santé (comme l'Allemagne, la Hollande et la Suisse) ont tous introduit ce que l'on appelle la péréquation des risques. La péréquation des risques a pour but d'éliminer tout intérêt des assureurs maladie à exploiter la sélection des risques. Il apparaît donc que la sélection des risques, le deuxième sujet mentionné dans le titre de ma conférence, soit un mécanisme à supprimer, parce que ayant une valeur négative pour l'ensemble de la société.

Pour éclairer les différents aspects de la sélection des risques, je commencerai par développer le concept de la sélection, en toute généralité, comme premier sujet de mon exposé. Très vite, nous verrons que le processus de sélection est étroitement lié à l'efficience économique. La même relation apparaît quand on passe de la sélection, comme concept général, à la sélection des risques dans le domaine de l'assurance. En effet, la sélection des risques s'avère être un réquisit à la réalisation d'un équilibre dans un marché d'assurance. La réfutation de ce résultat théorique en assurance maladie constitue une énigme que je ne résoudrai pas ce soir. En acceptant donc l'idée que la sélection des risques est à supprimer en assurance maladie, il se pose une question très pratique, à savoir: dans quelle mesure le succès des nouvelles formes de contrat, souvent appelées «managed care », est dû à la sélection des risques favorables? La seconde partie de ma conférence sera donc consacrée à la présentation d'une analyse économétrique qui vise à déterminer si les réductions des dépenses de santé réalisées par des variantes de «managed care » sont le résultat de la sélection des risques plutôt qu'une véritable innovation.

Ce texte reprend le contenu de la $23^{\text {ème }}$ conférence «Luigi Solari» prononcée à l'Université de Genève le 12 novembre 2002. 


\section{LA SÉLECTION: CONCEPT BIOLOGIQUE ET ÉCONOMIQUE}

Le concept de la sélection est étroitement lié au nom de Darwin (1871). La sélection assure la survie des populations les mieux adaptées aux exigences de leur environnement. En biologie, ces populations sont caractérisées par leurs gènes, qui sont égoïstes dans le sens qu'ils visent à maximiser leur chance de reproduction (Dawkins, 1976). Voilà donc l'idée de la maximisation sous contraintes, qui est si centrale en théorie économique, les contraintes étant celles imposées par un environnement naturel, dans le cas de la biologie.

Il y a toujours eu une application très directe du concept de la sélection en économie, quand il s'agit de la survie des entreprises. Sans barrières, ni à l'accès ni à la sortie du marché, seules les entreprises offrant un produit au coût unitaire minimum survivent. Dans ce cas, le critère de sélection exprime l'exigence de couverture des coûts de production par le prix de vente, imposée par l'environnement d'un marché ouvert. Pour Alchian (1950), la force de cette sélection économique est si grande que les choix productifs des entreprises pourraient même être aléatoires, car les entreprises qui évitent la faillite doivent avoir fait leur choix de façon approximativement optimale. Cette argumentation suggère que même des organisations qui n'ont pas pour but la maximisation du profit parviendront à minimiser les coûts de production, à cause de la pression de sélection exercée par la concurrence. Cette idée sera reprise plus tard, dans le contexte du comportement des assureurs maladie.

Le concept de sélection peut aussi être appliqué aux êtres humains pour expliquer leurs préférences. Par analogie avec l'argument précédant, on serait tenté de conclure que seuls les individus égoïstes, qui poursuivent leur propre avantage, survivent dans une économie de marché. Mais cette hypothèse a été modifiée à la lumière de la théorie des jeux. Considérons, d'une part, des individus agressifs qui poursuivent leur propre avantage à tout prix et, d'autre part, des individus qui sont disposés à coopérer. Quand deux individus agressifs se rencontrent, ils doivent dépenser beaucoup d'énergie pour se battre, ce qui limite le nombre de leurs descendants. Par contre, quand deux individus coopératifs interagissent, ils peuvent assurer leur survie à un moindre coût, ce qui engendre une progéniture plus grande. Bien sûr, il y a toujours les rencontres mixtes où les types agressifs l'emportent, mais comme le démontre Robert Frank (2000, ch. 7), il peut y avoir un équilibre stable qui contient une niche pour les individus coopératifs.

Passons maintenant du niveau des agents économiques individuels au niveau d'un marché, lieu de leur interaction. Le rôle important de la sélection peut alors être illustré par le modèle d'un marché concurrentiel simple. La figure 1 montre l'offre d'un bien homogène par unité de temps qui augmente avec le prix de vente, parce qu'à un prix plus élevé un nombre grandissant d'entreprises peut survivre. En revanche, la quantité demandée diminue en fonction du prix. Les intentions des vendeurs et des acheteurs sont concordantes quand les quantités offertes et demandées coïncident. Pour cela, le prix doit être au niveau $P^{e}$. Mais ce prix de marché sert aussi comme moyen de sélection. En effet, il sélectionne les consommateurs dont la disponibilité à payer est égale ou supérieure à $P^{e}$; eux seuls jouiront du bien en question. Par contre, les consommateurs qui ont une disponibilité à payer inférieure à $P^{e}$ seront contraints à renoncer au bien. D'autre part, les 
Figure 1: Marché concurrentiel interprété comme un mécanisme de sélection.

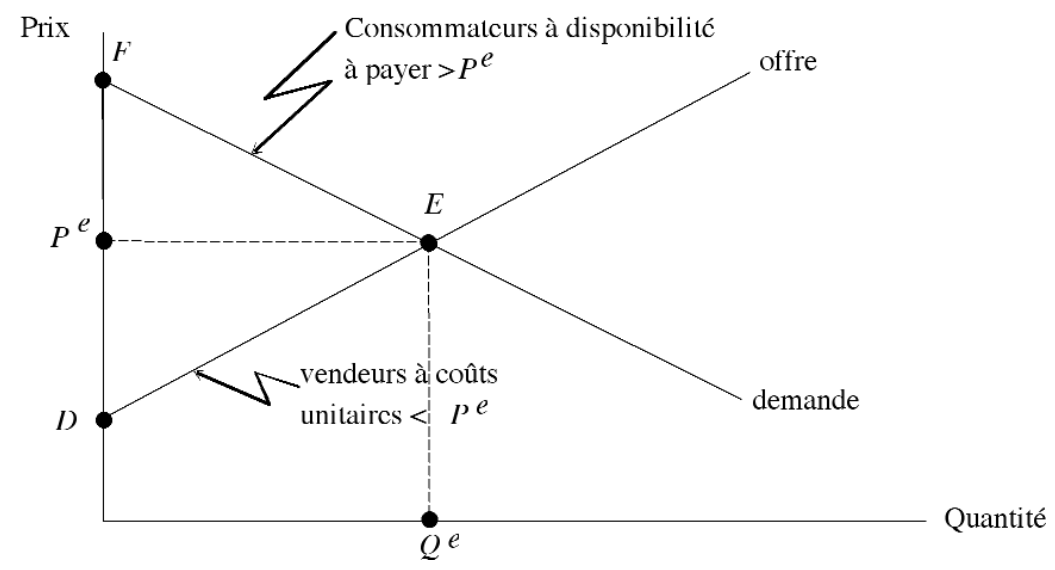

producteurs dont les coûts unitaires sont inférieurs au prix de vente $P^{e}$ seront sélectionnés comme vendeurs, alors que ceux qui affichent un coût unitaire supérieur à $P^{e}$ se verront exclus du marché ou contraints de le quitter.

Notons aussi la relation étroite entre le processus de sélection effectué par le prix de marché et l'efficience. Il est bien connu, qu'au prix $P^{e}$ le total des rentes des consommateurs et des producteurs est maximisé, ce qui traduit une condition d'efficience au sens de Pareto (Mas-Colell, Whinston, et Green, 1995, ch. 10F). Dans la figure 1, il s'agit de la somme des triangles $P^{e} E F$ et $P^{e} E D$. Par contre, si l'on n'était pas satisfait du résultat de cette sélection, on pourrait songer à baisser le prix pour favoriser les demandeurs. Mais cela causerait une réduction de la quantité offerte le long de $D E$, et la somme des deux superficies représentant les rentes des consommateurs et des producteurs diminuerait. La conséquence en serait une perte d'efficience dans le marché considéré.

Jusqu'à ce point, la sélection semble aboutir à une égalité de traitement des consommateurs, puisqu'ils paient le même prix de marché. Mais quand on quitte le monde de la concurrence parfaite, la sélection peut devenir un moyen de discrimination, ce qui la rend plus contestable. Prenons le cas d'un service lié à un réseau (distribution de gaz, d'électricité, chemin de fer, transport urbain), où l'on a typiquement un monopole naturel. Souvent, il est possible de mettre en place une régulation du prix de telle sorte que le prix du service soit égal à son coût marginal augmenté d'une charge d'accès permettant de couvrir les coûts totaux (Berg et Tschirhart, 1988).Bien entendu, il peut y avoir des coûts marginaux différents. Par exemple, un $\mathrm{kWh}$ mis à disposition vers midi, quand tous les réseaux de distribution sont déjà à la limite de leur capacité, a un coût marginal bien supérieur à celui d'un $\mathrm{kWh}$ livré vers minuit. De même, un passager additionnel d'un transport urbain n'exige presque jamais l'utilisation d'un véhicule supplémentaire quand il voyage en dehors des heures de pointe de trafic, mais il peut exiger une telle adaptation des véhicules en circulation, s'il veut voyager à une heure de pointe de trafic. 
Figure 2: Sélection des consommateurs comme condition d'efficience.

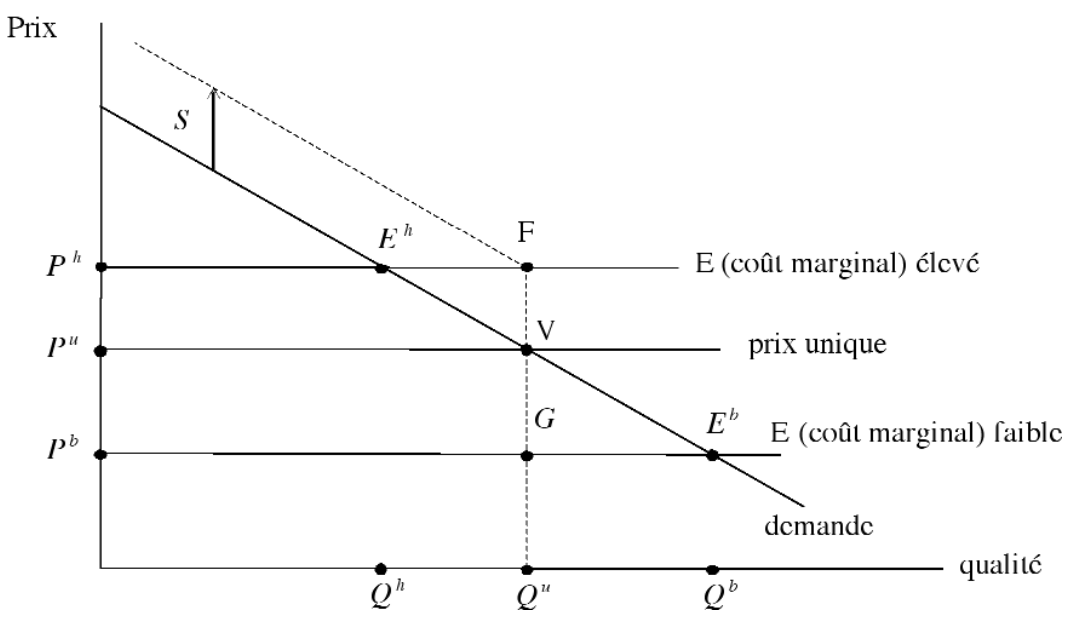

Dans la figure 2, on reconnaît un individu à coût marginal haut et un individu à coût marginal bas, en valeur espérée. Pour simplifier, ces deux individus ne se distinguent pas en tant que demandeurs. Ils ont donc la même disponibilité à payer pour une unité additionnelle du bien. Dans ce cas, l'efficience exige que la disponibilité à payer pour le service additionnel couvre le coût additionnel engendré. A l'individu à coût marginal élevé il faut donc facturer le prix haut $P^{h}$ et à l'individu à coût marginal bas, le prix bas $P^{b}$. Cette différenciation des prix suppose que le vendeur est capable de sélectionner ses clients. Quand le temps d'utilisation est étroitement lié au coût marginal du service, cette sélection est facile à réaliser. Dans d'autres circonstances, la sélection de la clientèle exige des efforts. Il faut souligner que le monopoleur considéré, pour des raisons d'efficience, mérite d'être encouragé à faire un tel effort de sélection.

Dans les marchés non parfaitement concurrentiels, la sélection est un réquisit à la différenciation des prix, qui est souvent jugée incompatible avec les notions de solidarité. La tentation pour les politiciens est alors d'octroyer un prix unique à ce monopole, par exemple le prix $P^{u}$. Mais l'imposition de la solidarité par la manipulation du prix a un coût social en termes de perte d'efficience. Premièrement, l'accroissement de la consommation de $Q^{h}$ à $Q^{u}$ pour l'individu dont le coût marginal est élevé intervient malgré que sa disponibilité à payer soit inférieure au coût marginal engendré. Deuxièmement, la consommation de l'individu dont le coût marginal est faible est réduite de $Q^{b}$ à $Q^{u}$ bien que sa disponibilité à payer couvre entièrement le coût marginal engendré. Dans la figure 2 , la première perte d'efficience correspond au triangle $V F E^{h}$, la deuxième, au triangle $V G E^{b}$. Il y a donc contradiction entre efficience et solidarité.

La solution proposée par les économistes pour atténuer ce conflit est bien connue. Si le prix $P^{u}$ est jugé acceptable, cela veut dire que le niveau de consommation $Q^{u}$ doit être atteint par tout le monde. Le point d'équilibre de l'individu à coût marginal élevé doit donc être $F$ plutôt que $E^{h}$. Mais si sa disponibilité à payer 
était plus marquée, cet individu choisirait le point $F$ tout en payant le prix haut $P^{h}$. Il s'agit donc d'accroître sa disponibilité à payer. Cela peut être réalisé par un subside (indiqué par $S$ dans la figure 2). C'est alors l'Etat qui devra effectuer une sélection pour éviter que des individus qui ont une disponibilité à payer suffisante touchent le subside. Ce problème peut être résolu parce que le revenu imposable constitue un indicateur de la disponibilité à payer. Mais il reste un autre problème. Comme le subside doit être financé par l'impôt, le conflit entre efficience et solidarité apparaît à nouveau, parce que la charge fiscale sur le revenu se répercute sur l'offre de travail et par là sur le revenu du travail. Les subsides ciblés ne font donc que atténuer, plutôt que résoudre, le conflit entre efficience et solidarité.

Pour conclure ce chapitre, retenons que les mécanismes de sélection s'avèrent être une condition nécessaire à l'efficience d'une économie de marché. De même, dans des conditions de monopole naturel (qui constitue l'autre extrême), des efforts de sélection sont toujours nécessaires pour réaliser l'efficience.

\section{LA SÉLECTION DES RISQUES}

Jusqu'ici, on a supposé que le vendeur peut distinguer les types de consommateurs. Mais quand ceux-ci se distinguent par leur tendance (c.-à-d. leur probabilité) à agir dans un certain sens, cette condition ne sera guère satisfaite. Notamment, les partenaires d'un contrat d'assurance se trouvent confrontés à de telles probabilités: l'assuré doit alors estimer la probabilité avec laquelle la compagnie d'assurance est capable de verser les prestations promises en cas de dommage. De plus, il y a toujours une probabilité que les prestations fassent défaut parce que le sinistre ne se conforme pas à la définition contractuelle. De son côté, l'assureur doit apprécier la probabilité avec laquelle un client subira des dommages.

De telles asymétries d'information existent dans beaucoup d'autres marchés. Le client qui souscrit un crédit hypothécaire doit estimer la probabilité avec laquelle il sera confronté à des conditions moins favorables dans l'avenir. La banque qui accorde ce crédit cherche à son tour à déterminer la probabilité avec laquelle le client sera capable de payer les intérêts. Dans le monde du travail, une nouvelle employée ambitieuse doit estimer si l'entreprise tiendra sa promesse de promotion future tandis que l'entreprise doit évaluer le risque que l'employée renoncera à sa carrière pour créer une famille.

Dans la littérature économique, on a traditionnellement mis l'accent sur l'asymétrie d'information au désavantage de l'entreprise. La raison probable en est que l'individu conclut un petit nombre de contrats d'un type donné, ce qui a pour effet qu'une asymétrie d'information défavorisant l'individu a peu d'effets systémiques. Les entreprises, elles, se spécialisent dans un petit nombre de contrats qu'elles concluent avec une grande fréquence. Une asymétrie d'information qui les défavorise aura donc un effet systémique dans l'économie qui mérite d'être analysé. Pour cette raison, on introduira un manque d'information du côté de l'entreprise, c.-à-d. de l'assureur. Mais pour commencer, admettons que l'assureur et le client détiennent une information parfaite.

Le modèle de référence est alors celui des biens conditionnels, développé par Arrow et Hirshleifer (Hirshleifer et Riley, 1979). Pour simplifier on ne distingue 
que deux états du monde: avec sinistre (qui se produit avec probabilité $\pi^{h}$ dans le cas d'un risque élevé et $\pi^{b}$ dans le cas d'un risque faible) et sans sinistre (avec probabilités $1-\pi^{h}$ et $1-\pi^{b}$ ). Pour un risque élevé, l'assureur doit donc payer une prestation $I$ avec probabilité $\pi^{h}$, ce qui donne une valeur espérée de $\pi^{h} I$. Si l'on admet des frais d'administration fixes $P_{0}$, la ligne de profit espéré nul est donnée par $P_{\mathrm{o}} K$ dans la figure 3 . La pente de cette ligne est $\pi^{h}$. Pour un risque faible, la ligne de profit espéré nul est représentée par $P_{0} C$, avec une pente $\pi^{b}$. Comme toujours, les préférences de l'individu sont reflétées par des courbes d'indifférence, dont le gradient est orienté sud-est, parce que la prime est un mal plutôt qu'un bien. Or, ces courbes d'indifférence ont deux particularités (Hirshleifer et Riley, 1979).

1. Leur pente est égale à $\pi^{h}\left(\pi^{b}\right.$ respectivement) là où la prestation d'assurance est égale à la perte.

2. Leur courbure reflète le degré d'aversion au risque de l'individu. Une courbure plus marquée correspond à un degré plus important d'aversion au risque.

Figure 3: Sélection des risques comme condition d'efficience.

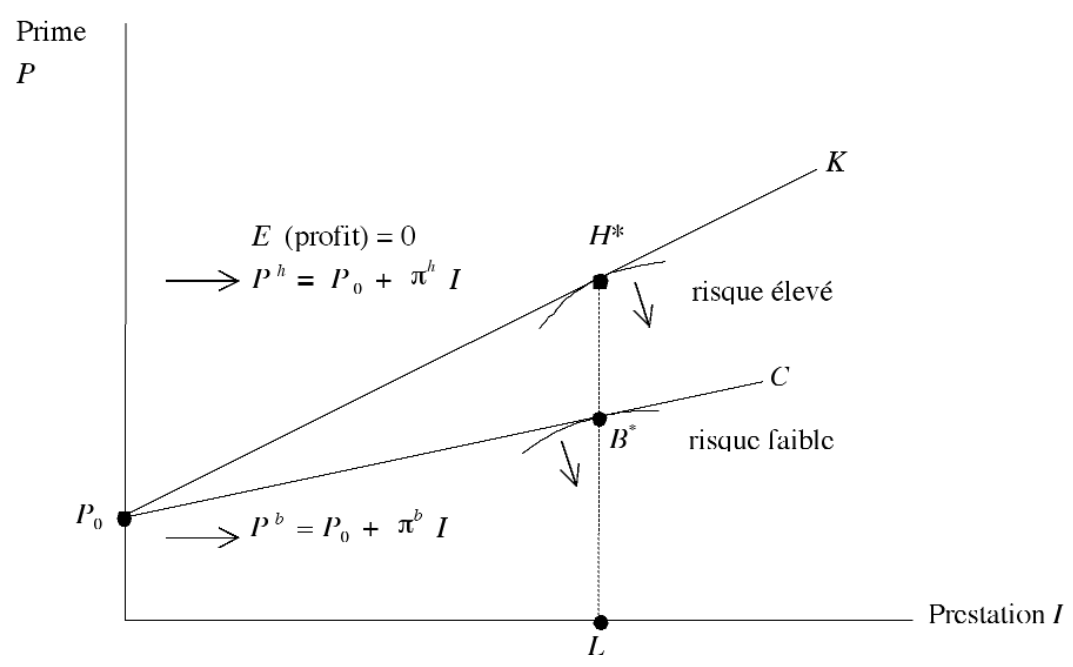

La première caractéristique est d'une importance particulière parce qu'elle permet de distinguer les types de risques. En effet, les risques élevés sont caractérisés par des courbes d'indifférence à pente élevée, tandis que les risques faibles sont caractérisés par des courbes d'indifférence à pente faible. Lorsque l'assureur peut sélectionner les deux types de risque, il offre des contrats sur $P_{\mathrm{o}} K$ aux risques élevés mais sur $P_{0} C$ aux risques faibles. Les conditions de tangence pour un optimum sont alors satisfaites aux points $H^{*}$ et $B^{*}$. Ainsi l'équilibre est caractérisé par la couverture complète du risque, mais à prime différenciée. Cet équilibre est aussi efficient au sens de Pareto. En effet, une prime moyenne pour les deux risques ne serait pas soutenable parce qu'un assureur concurrent pourrait offrir 
des conditions plus favorables aux risques faibles. L'assureur ainsi concurrencé aurait à payer des prestations pour des risques élevés tandis que ses primes seraient calculées sur la base d'un mélange contenant aussi des risques faibles. Sa faillite ne se ferait pas attendre, et l'équilibre efficient serait perdu.

En somme, la sélection des risques est nécessaire pour garantir l'efficience. Cela a l'air de contredire la solidarité. En fait, il y a solidarité, car ceux qui ne subissent pas de perte paient leur prime pour financer ceux qui ont subi un sinistre. Mais c'est une solidarité au regard du hasard, qui ne comporte pas de redistribution systématique. Une telle redistribution pourrait être réalisée par une prime moyenne pour tous les risques. Cependant, on vient de voir que tout contrat qui mélangerait les deux types de risques causerait une perte d'efficience.

Supposons maintenant qu'il y a asymétrie d'information au détriment de l'assureur. L'initiative réside alors chez les assurés. La figure 3 montre que les risques élevés ont tout intérêt à se présenter comme des risques faibles pour obtenir les conditions de contrat qui étaient réservées aux risques faibles, soit la ligne $P_{0} C$. Mais la ligne $P_{\mathrm{o}} C$ définit les combinaisons de primes et prestations assurant un profit espéré nul, à condition qu'il s'agisse de risques faibles. La survie économique de l'assureur visé serait en danger. Sa faillite s'ensuivrait, et les risques faibles seraient de nouveau à la recherche d'un contrat favorable mais guère durable.

Figure 4 : Prime unique et la «chasse aux bons risques ».

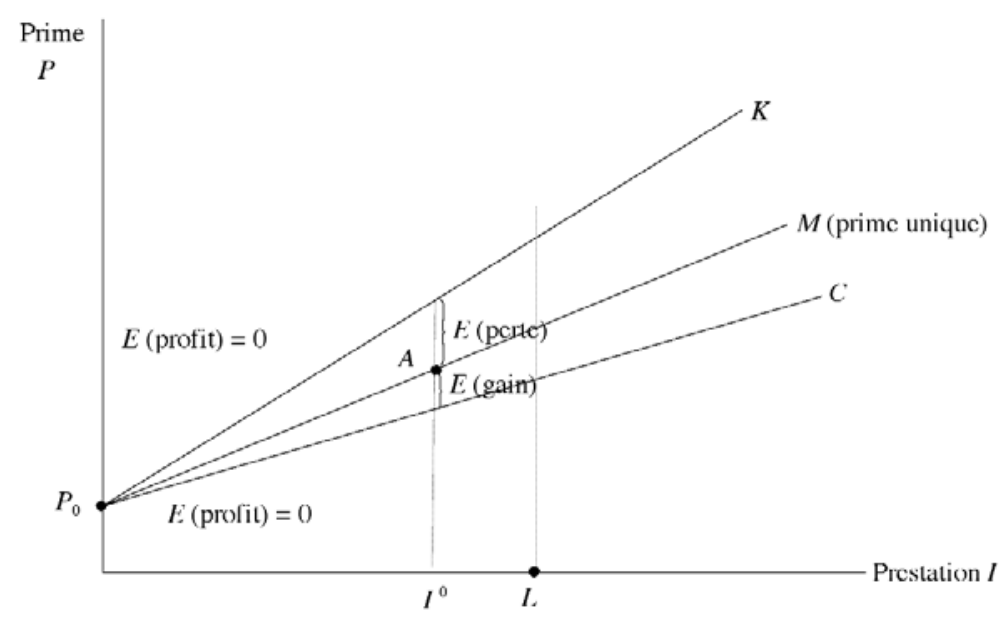

On peut conclure que l'existence même d'un équilibre est en jeu à cause d'une telle asymétrie d'information. Pour apporter de la stabilité au marché, les assureurs devraient donc faire des efforts continus pour estimer ces probabilités $\pi^{h}$ et $\pi^{b}$ d'un sinistre. Une fois de plus, la sélection (ici celle des risques) s'avère être une condition nécessaire à l'efficience économique. Si la prime d'un risque élevé est trop importante pour les économiquement faibles, l'Etat est appelé à subventionner cette prime à titre individuel, comme dans le cas du monopole naturel. 
Il est très intéressant de constater qu'il existe des branches d'assurance où la sélection des risques est acceptée, peut-être parce que sa relation avec l'efficience est suffisamment évidente. Dans l'assurance-vie privée, personne n'est étonné par le fait que les individus qui concluent un contrat à un âge où le risque de mort est déjà élevé doivent payer une prime supérieure à ceux dont l'âge implique un risque de mort plus faible. En assurance-automobile, les conducteurs qui font courir des risques élevés doivent payer un multiple de la prime de base.

\section{LE CAS \\ DE L'ASSURANCE MALADIE}

Les choses sont différentes en assurance maladie sociale. En Suisse on a connu une régulation des primes dès la loi de 1911. Pour encourager la souscription d'une couverture par les jeunes, la prime était fixée en fonction de l'âge auquel le contrat était conclu. De plus, il y avait une surcharge générale de $10 \%$ pour les femmes. Quant à la Confédération, elle versait des subventions aux caissesmaladie calculées sur la base d'un forfait par personne assurée.

Avec la nouvelle loi de 1994, la Confédération verse des subsides à titre personnel, complétés par les Cantons. Tous ceux pour qui la prime représente plus de $10 \%$ de leur revenu imposable (même $8 \%$ dans quelques Cantons plus généreux) ont droit à un subside. On reconnaît facilement une augmentation de la disponibilité à payer pour le bien «couverture d'assurance maladie» décrite plus haut. Rien n'aurait alors empêché l'installation d'un régime concurrentiel, avec une sélection des risques impliquant des contrats à primes différenciées. Comme on vient de voir, cela serait dans l'intérêt de l'efficience économique. En réalité, le législateur a décidé tout autrement, à savoir: d'introduire une prime unique pour tous les adultes.

La conséquence de cette régulation ressort de la figure 4. Rappelons que les deux lignes droites $P_{\mathrm{o}} K$ et $P_{\mathrm{o}} C$ définissent des combinaisons primes-prestations qui garantissent un profit espéré nul. Les deux types de risque continuent d'exister, mais l'assureur est maintenant contraint de calculer une prime moyenne unique. Les prestations sont aussi prescrites; elles sont représentées par le montant $I^{\circ}$ qui correspond au contrat $A$. Mais s'il s'agit d'un risque élevé, ce point $A$ signale une prime trop basse, qui cause une perte de la valeur espérée. Par contre, s'il s'agit d'un risque faible, l'assureur réalise un gain en valeur espérée. La prime unique impose à l'assureur d'avoir un nombre suffisant de risques faibles pour assurer sa survie et cela indépendamment de son objectif de profit ou non.

Alors que les efforts destinés à sélectionner les risques servaient à établir un équilibre efficient dans l'analyse précédente, ces efforts sont maintenant dénoncés comme une «chasse aux bons risques». Cette dénonciation est d'autant plus marquée que le législateur attend de la concurrence des innovations qui aideraient à maîtriser les dépenses de santé. Or, plutôt que de se lancer dans des négociations difficiles avec les médecins, chefs d'hôpitaux et pharmaciens pour faire baisser le coût de leurs services, les assureurs maladie se livrent à cette chasse (Van de Ven et Ellis, 2000). Pour orienter leurs efforts dans la bonne direction, on a installé, avant même l'entrée en vigueur de la nouvelle loi, la péréquation des risques. Il s'agit d'une régulation secondaire qui devient nécessaire pour neutraliser la 
tendance à la «chasse aux bons risques », suscitée par la régulation primaire des primes.

Le mécanisme de la péréquation des risques sanctionne l'assureur qui affiche une structure de la population assurée plus favorable que la moyenne. Il doit payer une contribution au fonds de péréquation. En revanche, un assureur qui prend en charge une part de risques élevés plus importante que la moyenne reçoit un subside de la part du fonds. Or, un tel mécanisme est toujours basé sur des indicateurs observables. Actuellement, il s'agit de l'âge et du sexe, quoique ces deux variables n'expliquent que 3\% de la variance des dépenses annuelles (Van de Ven et Ellis, 2000). Par conséquent, les assureurs continuent de tirer profit des indicateurs qu'ils dérivent de leurs systèmes d'information internes, en particulier de ceux qui concernent le développement et la composition des dépenses de santé dans le passé.

Dans cette situation, toute innovation de produit est soupçonnée de contribuer à la «chasse aux bons risques». C'est aussi le cas avec les nouveaux contrats dit de «managed care». A Zurich nous avons eu la chance de coopérer avec un assureur-maladie qui offre un contrat conventionnel, avec paiement à l'acte des médecins de ville, et trois variantes connues de «managed care» (tableau 1). Comme

Tableau 1: Les contrats analysés.

\begin{tabular}{|l|l|}
\hline Conventionnel & Paiement à l'acte \\
\hline HMO & $\begin{array}{l}\text { Paiement forfaitaire; centres de traitement réunissant plusieurs médecins; } \\
\text { surveillance mutuelle }\end{array}$ \\
\hline IPA & $\begin{array}{l}\text { Réseau de médecins indépendants dans lequel les omnipraticiens surveillent } \\
\text { la filière des traitements; paiement à l'acte comme règle }\end{array}$ \\
\hline PPO & $\begin{array}{l}\text { L'assuré garde la liberté du choix dans une liste de médecins qui exclut les } \\
\text { plus coûteux }\end{array}$ \\
\hline Seuls sont retenus les assurés ayant la franchise minimale de 230 Frs et âgés de 26 ans et plus
\end{tabular}

ces variantes ont été développées aux Etats-Unis, on retient ici les acronymes américains.

En 2000, la grande majorité (86\%) de la clientèle de cet assureur est restée avec le contrat conventionnel. Toutefois, le 6\% a opté pour la HMO (Health Maintenance Organization). La HMO connaît un paiement forfaitaire des médecins, qui travaillent dans des centres de traitement réunissant jusqu'à une douzaine d'omnipraticiens et spécialistes. Le paiement forfaitaire change fortement les incitations des médecins conventionnés, car ils doivent payer tous les services hors réseau par leur propre budget. Pour assurer la qualité de leurs traitements, ils pratiquent aussi une surveillance mutuelle.

La IPA (Independent Practice Association) consiste en un réseau de médecins indépendants. Les assurés doivent d'abord contacter un omnipraticien, qui surveille la filière des traitements. Comme les médecins continuent d'être payés à l'acte, ils ont moins intérêt à maîtriser les dépenses de santé. Enfin, cet assureur- 
maladie gère aussi une PPO (Preferred Provider Organization). La PPO est basée sur une liste de médecins qui exclut les praticiens les plus coûteux. Les assurés gardent leur liberté de choix et les médecins sont payés à l'acte.

$\mathrm{Au}$ cours des quatre dernières années, mon assistant Hansjörg Lehmann a comparé les dépenses de santé de ces quatre modalités de prise en charge. Il a trouvé, notamment, une réduction de $62 \%$ des dépenses de santé dans le cadre de la HMO, comparé au contrat conventionnel. Une telle différence nourrit le soupçon que l'assureur ait pu attirer des risques faibles par son offre HMO et par les offres «managed care», plus généralement. M. Lehmann vient d'achever une excellente thèse de doctorat qui cherche à établir la part de cette différence de dépenses qui résulte de la sélection des risques. Le reste serait attribuable à la modification des incitations médicales, à la surveillance mutuelle des médecins ou à la meilleure gestion des filières de traitement, bref à l'innovation. Mais il faut signaler d'emblée, que les effets de sélection qui conduisent à une migration vers l'assureur étudié aussi bien qu'à des migrations de cet assureur vers un concurrent n'ont pas pu être pris en compte. Pour homogénéiser l'échantillon, seuls les assurés ayant la franchise minimale de CHF 230 et âgés de 26 ans et plus ont été retenus.

La nouveauté de l'analyse de M. Lehmann réside dans le fait qu'elle est basée sur des observations répétées. L'hypothèse de base postule que c'est l'état de santé qui influence de façon décisive les dépenses de santé. Ainsi, un assureur visant les risques faibles tenterait d'estimer, à partir des caractéristiques observables des assurés une variable non observable nommée «bonne santé latente», qui explique pourquoi les dépenses de santé restent au-dessous de leur valeur espérée pendant une série d'années. Pour simuler cette stratégie (approche «L»), on utilise quelques 60000 observations de la période 1997-1999 pour expliquer les dépenses de santé en fonction des caractéristiques individuelles et du type de contrat, par le biais d'une régression qui donne des résidus $\hat{\epsilon}$ (figure 5). La régression doit être du type Tobit, pour rendre compte du fait que certains des assurés ont une dépense

Figure 5: Approche L; mesure des effets de sélection par la santé latente.

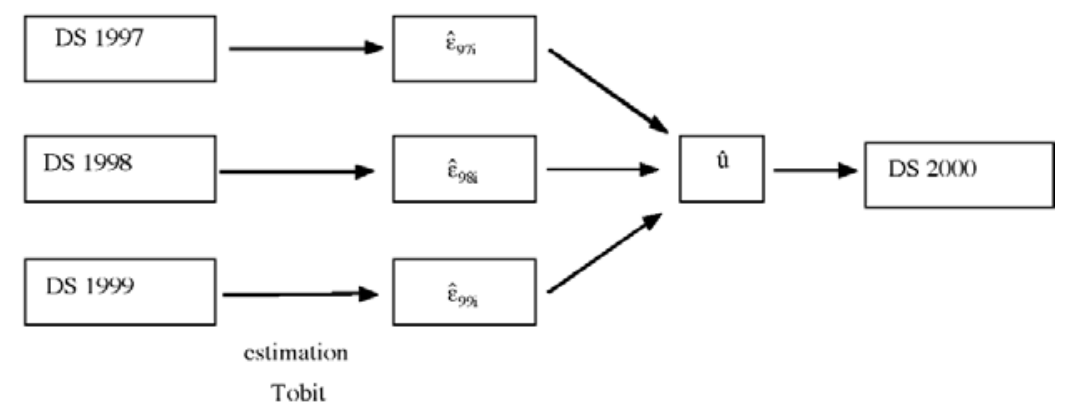

DS: dépenses de santé

de santé nulle. La moyenne de ces trois $\hat{\epsilon}$ devrait constituer une assez bonne estimation de la santé latente traduisant les effets de la sélection des risques sur les dépenses de santé de l'an 2000. 
Pour donner quelques détails, on suppose que les résidus de la régression Tobit contiennent deux composantes:

$$
\epsilon_{i t}=u_{i}+\xi_{i t}
$$

où $u_{i}$ représente la composante individuelle, qui ne varie pas dans le temps, tandis que $\xi_{i t}$ est une composante générale qui varie parmi les individus et dans le temps. On identifie donc $u_{i}$ avec l'état de santé latent d'un individu. L'estimation du modèle Tobit ne permet pas d'évaluer les composantes $u_{i}$ pour chaque assuré. Elle ne permet d'estimer que la variance de cette composante. Toutefois, on peut construire un indicateur des résidus $\hat{u}_{i}$ en calculant la moyenne arithmétique des trois résidus combinés, soit:

$$
\hat{u}_{i}=\frac{\hat{\epsilon}_{i 97}+\hat{\epsilon}_{i 98}+\hat{\epsilon}_{i 99}}{3}=\hat{\mathrm{E}}\left(\epsilon_{i t} \mathrm{li}\right)
$$

Pour faciliter les comparaisons avec des études américaines, on a modélisé séparément, d'une part, l'initialisation d'un traitement médical et, d'autre part, les dépenses de santé étant donnée l'initialisation. Le tableau 2 montre deux estimations Probit de la probabilité d'initialisation, sans et avec santé latente. Sans l'indicateur de santé latente $\hat{u}$, les assurés HMO semblent être caractérisés par une probabilité d'initialisation qui est 7.3 points de pourcentage au-dessous de celle des assurés ayant un contrat conventionnel. Par contre, les modalités IPA et PPO se distingue-

\begin{tabular}{|c|c|c|c|c|c|c|c|}
\hline \multirow[t]{2}{*}{ Variables } & \multirow{2}{*}{$\begin{array}{l}\text { Signe } \\
\text { attendu }\end{array}$} & \multicolumn{3}{|c|}{ Estimation sans santé latente } & \multicolumn{3}{|c|}{ Estimation avec santé latente } \\
\hline & & Coefficient & Ecart-type & $\begin{array}{l}\text { Variation } \\
\text { en } \%\end{array}$ & Coefficient & Ecart-type & $\begin{array}{l}\text { Variation } \\
\text { en } \%\end{array}$ \\
\hline HMO & - & $-0.248 * * *$ & 0.024 & -7.3 & $-0.239 * * *$ & 0.027 & -5.4 \\
\hline IPA & - & $0.074 * * *$ & 0.023 & 1.9 & 0.036 & 0.026 & 0.8 \\
\hline $\mathrm{PPO}$ & - & $0.174 *$ & 0.068 & 4.3 & 0.072 & 0.076 & 1.5 \\
\hline AGE & + & $0.009 * * *$ & 0.002 & $2.5 \mathrm{a})$ & $0.016^{* * * *}$ & 0.003 & $3.4 a)$ \\
\hline $\mathrm{AGE}^{2}$ & + & $0.001 * * *$ & 0.000 & $0.3 \mathrm{a})$ & $0.001 * *$ & 0.000 & $0.02 a)$ \\
\hline SEX_M & - & $-0.413 * * *$ & 0.012 & -11.3 & $-0.436 * * *$ & 0.014 & -9.6 \\
\hline REGION_U & + & $0.072 * * *$ & 0.015 & 2.0 & $0.070 * * *$ & 0.017 & 1.5 \\
\hline SUBSIDE & & 0.009 & 0.014 & 0.3 & -0.008 & 0.017 & -0.2 \\
\hline$\hat{\mathrm{u}}$ & + & & & & $0.255^{* * *}$ & 0.002 & 5.4 \\
\hline Constante & & 0.132 & 0.076 & & 0.076 & 0.088 & \\
\hline & & \multicolumn{3}{|c|}{$\begin{array}{l}\text { Nombre d'observations }=59 \text { ' } 695 \\
\text { LR }: \chi(18)=5,197 ; \text { Prob }>\chi=0.0000 \\
\text { Pseudo R2 }=0.09\end{array}$} & \multicolumn{3}{|c|}{$\begin{array}{l}\text { Nombre d'observations }=599^{\prime} 695 \\
\text { LR }: \chi(19)=20,785 ; \text { Prob }>\chi=0.0000 \\
\text { Pseudo R2 }=0.34\end{array}$} \\
\hline \multicolumn{8}{|c|}{$\begin{array}{l}\text { HMO, IPA, PPO = } 1 \text { si l'assuré a choisi la modalité correspondante } ;=0 \text { sinon. } \\
\text { SEXE_M = } 1 \text { si l'assuré est de sexe masculin } ;=0 \text { sinon. } \\
\text { REGION_U = } 1 \text { si le domicile de l'assuré est en zone urbaine } ;=0 \text { sinon. } \\
\text { SUBSIDE = } 1 \text { si l'assuré reçoit un subside de prime } ;=0 \text { sinon. } \\
\text { û = indicateur de santé latente selon la définition (2). }\end{array}$} \\
\hline
\end{tabular}

Tableau 2: Approche L; modèle Probit de l'initialisation d'un traitement en 2000. 
raient par une probabilité plus marquée que leur contrepartie conventionnelle. Cette différence disparaît quand la régression Probit est complétée par la santé latente. Il reste uniquement une différence de 5 points de pourcentage en faveur de l'HMO, soit un tiers de la différence brute observée, qui représente 15 points de pourcentage.

Conditionnellement à l'initialisation, on peut analyser les dépenses (après transformation logarithmique) par la méthode des moindres carrées (Tableau 3). Sans la variable représentant la santé latente, les assurés HMO auraient des dépenses de $42 \%$ inférieures à celles des assurés avec contrat conventionnel. Les effets de la PPO (liste restreinte de médecins) et de la IPA (réseau de médecins indépendants) seraient moins marqués. Quand la santé latente est ajoutée à la Tableau 3: Approche $L$; estimation des moindres carrés du modèle des logarithmes des dépenses de santé en 2000 (initialisation du traitement médical connue).

\begin{tabular}{|c|c|c|c|c|c|c|c|}
\hline \multirow[t]{2}{*}{ Variables } & \multirow{2}{*}{$\begin{array}{l}\text { Signe } \\
\text { attendu }\end{array}$} & \multicolumn{3}{|c|}{ Estimation sans santé latente } & \multicolumn{3}{|c|}{ Estimation avec santé latente } \\
\hline & & Coefficient & Ecart-type & $\begin{array}{l}\text { Variation } \\
\text { en \% }\end{array}$ & Coefficient & Ecart-type & $\begin{array}{l}\text { Variation } \\
\text { en } \%\end{array}$ \\
\hline THMO & - & $-0.547 * * *$ & 0.032 & -42.1 & $-0.462 * * *$ & 0.029 & -36.8 \\
\hline IPA & - & $-0.125^{* * * *}$ & 0.025 & -11.7 & $-0.101 * * *$ & 0.022 & -9.6 \\
\hline PPO & - & $-0.289 * * *$ & 0.069 & -25.1 & $-0.244 * * *$ & 0.063 & -21.3 \\
\hline AGE & + & $-0.009 * * *$ & 0.002 & $-9.1^{\text {a) }}$ & $-0.008 * *$ & 0.002 & $7.7^{\mathrm{a})}$ \\
\hline $\mathbf{A G E}^{2}$ & + & $0.001 * * *$ & 0.000 & $0.3^{\mathrm{a})}$ & $0.001 * * *$ & 0.000 & $0.3^{\mathrm{a})}$ \\
\hline SEX_M & - & $-0.102 * * *$ & 0.013 & -9.7 & $-0.237 * * *$ & 0.012 & -21.1 \\
\hline REGION_U & + & $0.097 * * *$ & 0.016 & 10.2 & $0.106^{* * *}$ & 0.015 & 11.2 \\
\hline SUBSIDE & & 0.012 & 0.016 & 1.2 & 0.004 & 0.014 & 0.4 \\
\hline$\hat{\mathbf{u}}$ & + & & & & $0.245^{* * *}$ & 0.002 & 0.25 \\
\hline Constante & & $6.656 * * *$ & 0.083 & & $6.327 * * *$ & 0.075 & \\
\hline & & \multicolumn{3}{|c|}{$\begin{array}{l}\text { Nombre d'observations = } 47^{\prime} 189 \\
\mathrm{~F}\left(18,477^{\prime} 189\right)=397 \\
\text { Prob>F }=0.0000 \\
\mathrm{R}^{2} \text { ajusté }=0.13\end{array}$} & \multicolumn{3}{|c|}{$\begin{array}{l}\text { Nombre d'observations = } 47^{\prime} 189 \\
\mathrm{~F}\left(19,477^{\prime} 189\right)=989 ; \\
\text { Prob>F }=0.0000 \\
\mathrm{R}^{2} \text { ajusté }=0.29\end{array}$} \\
\hline \multicolumn{8}{|c|}{$\begin{array}{l}* \mathrm{p} \leq 0.05, * * \mathrm{p} \leq 0.01, * * * \mathrm{p} \leq 0.001 \\
\text { a)Calculée pour une période de } 10 \mathrm{ans}\end{array}$} \\
\hline \multicolumn{8}{|c|}{$\begin{array}{l}\text { Remarque: } \\
\text { HMO, IPA, PPO = } 1 \text { si l'assuré a choisi la modalité correspondante; }=0 \text { sinon. } \\
\text { SEXE_M = } 1 \text { si l'assuré est de sexe masculin; = } 0 \text { sinon. } \\
\text { REGION_U = } 1 \text { si le domicile de l'assuré est en zone urbaine; = } 0 \text { sinon. } \\
\text { SUBSIDE }=1 \text { si l'assuré reçoit un subside de prime; }=0 \text { sinon. } \\
\text { û = indicateur de santé latente selon la définition (2). }\end{array}$} \\
\hline
\end{tabular}

régression, tous les trois effets sont réduits. Toutefois, la modalité HMO reste en premier rang avec une différence des dépenses de presque $37 \%$, suivie par la PPO avec $21 \%$ et la IPA avec quelque $10 \%$.

En conclusion, l'approche $L$, qui cherche à neutraliser les effets de sélection par le biais d'un indicateur de la santé latente des assurés, aboutit à des effets «managed care» plus modestes sans pourtant les effacer. 
Figure 6: Approche $C$; mesure des effets de sélection par le choix du contrat en 2000.

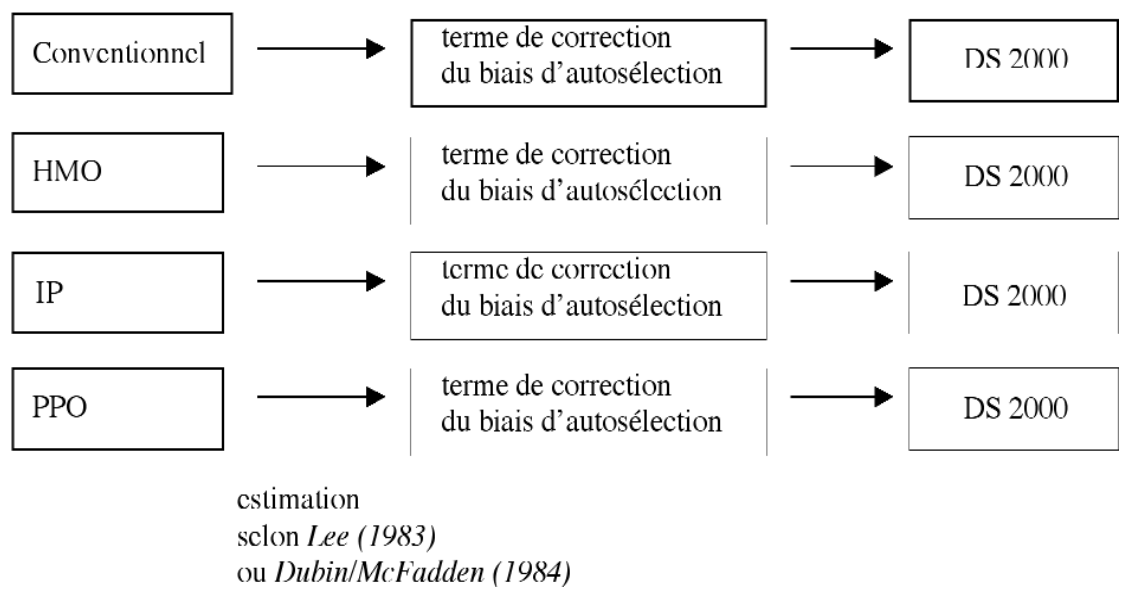

Mais il existe une autre approche économétrique du problème de sélection. L'approche $« C »$ (contrat) considère le choix du contrat comme un mécanisme qui auto-sélectionne les assurés de telle sorte que ceux qui ont choisi une variante donnée de contrat ne sont pas représentatifs de la population totale des assurés (figure 6).

Cette approche est plus traditionnelle, parce qu'elle ne requiert que des observations d'une même année pour expliquer le choix du contrat. Dans notre cas, nous avons modélisé d'abord le choix entre le contrat conventionnel et une alternative de type «managed care», puis le choix de l'alternative «managed care» parmi les trois alternatives offertes (HMO, IPA, PPO) pour les assurés ayant opté pour ce type de contrat. La modélisation de la probabilité de choix de la variante contractuelle, $\hat{P}_{s}$, a été réalisée à l'aide d'un modèle de type Logit conditionnel. Pour chaque estimation, on peut construire un terme de correction du biais d'autosélection, $f_{s}\left(\hat{P}_{s}\right)$, afin de permettre une estimation convergente de la régression des dépenses de santé $2000\left(D S_{s}\right)$, par la relation suivante:

$$
D S_{s}=X_{s} \beta_{s}+\lambda_{s} f_{s}\left(\hat{P}_{s}\right)+\eta_{s},
$$

avec

$X_{s} \beta_{s} \quad$ autres variables explicatives avec leurs coefficients

$\lambda_{s} \quad$ coefficient reflétant l'importance du mécanisme d'auto-sélection

$f_{s}($.$) \quad transformation similaire à l'inverse du ratio de Mills$

$\eta_{s} \quad$ variable aléatoire centrée.

Il s'agit là d'une généralisation de la méthode d'estimation des modèles Tobit proposée par Heckman (1979), introduite par Lee (1983) et par Dubin et McFadden (1984), notamment. Comme la solution de Lee (1983) est moins compliquée, c'est elle qui a été retenue.

Le terme de correction du biais d'auto-sélection est statistiquement significatif dans toutes les quatre équations des dépenses de santé. La prise en compte du choix du contrat s'avère donc importante (tableau 4). Les valeurs $t$ négatives des 
Tableau 4: Approche $C$ et comparaison avec l'approche $L$.

\begin{tabular}{|c|c|c|}
\hline \multicolumn{3}{|c|}{ (a) Significativité statistique des termes de correction $\lambda_{\mathrm{s}}$ dans 4 équations pour DS } \\
\hline $\begin{array}{l}\text { Conventionnel } \\
\text { HMO } \\
\text { IPA } \\
\text { PPO }\end{array}$ & $\begin{array}{l}\mathrm{t}=2.06 \\
\mathrm{t}=-2.08 \\
\mathrm{t}=3.10 \\
\mathrm{t}=-2.00\end{array}$ & $\begin{array}{l}P>t=0.039 \\
P>t=0.038 \\
P>t=0.002 \\
P>t=0.046\end{array}$ \\
\hline \multicolumn{3}{|c|}{ (b) Significativité statistique des termes de correction $\lambda_{s}$ après insertion de la santé latente $\hat{u}$} \\
\hline $\begin{array}{l}\text { Conventionnel } \\
\text { HMO } \\
\text { IPA } \\
\text { PPO }\end{array}$ & $\begin{array}{l}t=0.05 \\
t=0.18 \\
t=1.70 \\
t=2.00\end{array}$ & $\begin{array}{l}\mathrm{P}>\mathrm{t}=0.958 \\
\mathrm{P}>\mathrm{t}=0.854 \\
\mathrm{P}>\mathrm{t}=0.089 \\
\mathrm{P}>\mathrm{t}=0.046\end{array}$ \\
\hline
\end{tabular}

variantes HMO et PPO indiquent que les dépenses de santé y sont inférieures grâce au choix fait en faveur de ces deux alternatives. Ce choix est à son tour influencé par les caractéristiques de l'assuré, parmi elles sa santé inobservée. La partie (a) du tableau 4 suggère donc que la sélection des risques opérant par le biais du choix des contrats a une influence importante sur les dépenses de santé.

Maintenant on peut comparer les deux approches en insérant dans les équations des dépenses de santé les termes de correction du biais d'auto-sélection (approche $C$ ) ainsi que le résidu moyen comme indicateur de santé latente (approche $L$ ). Lorsque les équations des dépenses de santé sont complétées de cette façon, les termes de correction perdent leur signification statistique dans trois cas sur quatre (partie b) du tableau 4). Par contre, la variable $\hat{u}$ (santé latente) est toujours significative. L'approche $L$ semble donc être plus informative que l'approche $C$. Tout autre résultat aurait été surprenant car l'approche $L$ utilise les observations des trois années précédentes pour estimer un indicateur de la santé latente, tandis que l'approche $C$ n'utilise que les observations de l'année 2000.

Néanmoins, les deux approches arrivent à des conclusions très similaires. Après insertion de la santé latente, l'approche $L$ indique que les membres de la HMO réalisent des dépenses de santé qui sont de $37 \%$ inférieures à celles des assurés ayant souscrit un contrat conventionnel. Dans le cadre de l'approche $C$, ce chiffre, calculé sur la base des valeurs moyennes des variables explicatives figurant dans les équations «contrat conventionnel» et «contrat $\mathrm{HMO}$ », est de 40\%

Tableau 5: Comparaison des résultats.

\begin{tabular}{|l|l|l|}
\hline \multicolumn{3}{|l|}{ Réduction des DS en pourcent après correction pour les effets de sélection } \\
\hline Base: & Approche $L$ & Approche $C$ \\
Contrat conventionnel & (v. graphique 8) & (selon Lee, 1983) \\
\hline HMO & $-36.8 * * *$ & $-40.1 * * *$ \\
IPA & $-9.6 * * *$ & $-10.9 * * *$ \\
PPO & $-21.3 * * *$ & $-22.8 * * *$ \\
\hline
\end{tabular}

(tableau 5). Les différences sont encore plus modestes dans les cas de l'IPA et de la PPO. En somme, on ne devrait pas commettre d'erreurs graves en utilisant les estimations de l'approche $L$ pour calculer les effets liés à la sélection des risques et ceux liés à l'innovation que présentent les contrats du type «managed care». 
Ce calcul est effectué dans le tableau 6. Dans le cas de la HMO, on part d'une différence brute par rapport à la médecine conventionnelle de $62 \%$. Les données montrent aussi que les membres HMO qui ont commencé au moins un traitement pendant l'an 2000 ont des dépenses de santé inférieures de 53\%. L'effet coût causé par une fréquence réduite d'initialisations est ainsi de 9\%. Maintenant, ce chiffre doit être réparti entre les effets «innovation» et «sélection des risques». Dans le tableau 2, on a estimé à 5\% l'effet lié à la HMO, après insertion de la santé latente dans l'équation. La différence de probabilité d'initialisation observée est de $15 \%$

Tableau 6: Estimation des effets d'innovation et de sélection sur les dépenses de santé en 2000.

\begin{tabular}{|c|c|c|c|c|c|c|c|c|c|}
\hline & \multicolumn{3}{|l|}{ HMO } & \multicolumn{3}{|l|}{ IPA } & \multicolumn{3}{|l|}{ PPO } \\
\hline & $\begin{array}{l}\text { Total } \\
\%\end{array}$ & $\begin{array}{l}\text { Innova- } \\
\text { tion } \\
\%\end{array}$ & $\begin{array}{l}\text { Sélec- } \\
\text { tion } \\
\%\end{array}$ & Total & $\begin{array}{l}\text { Innova- } \\
\text { tion } \\
\%\end{array}$ & \begin{tabular}{|l|} 
Sélec- \\
tion \\
$\%$
\end{tabular} & $\begin{array}{l}\text { Total } \\
\%\end{array}$ & $\begin{array}{l}\text { Innova- } \\
\text { tion } \\
\%\end{array}$ & $\begin{array}{l}\text { Sélec- } \\
\text { tion } \\
\%\end{array}$ \\
\hline $\begin{array}{l}\text { A Différence } \\
\text { brute }\end{array}$ & -62 & & & -34 & & & -39 & & \\
\hline $\begin{array}{l}\text { B Différence après } \\
\text { initialisation }\end{array}$ & -53 & & & -34 & & & -39 & & \\
\hline $\begin{array}{l}\text { C Différence due à } \\
\text { l'initialisation }\end{array}$ & -9 & & & 0 & & & 0 & & \\
\hline $\begin{array}{l}\text { D Répartition } \\
\text { de } C^{a)}\end{array}$ & & -3 & -6 & & 0 & 0 & & 0 & 0 \\
\hline $\begin{array}{l}\text { E Répartition } \\
\text { de B }\end{array}$ & & -37 & -16 & & -10 & -24 & & -21 & -18 \\
\hline $\begin{array}{c}\text { F Répartition } \\
\text { de } \mathrm{B}+\mathrm{C}\end{array}$ & & -40 & -22 & & -10 & -24 & & -21 & -18 \\
\hline
\end{tabular}

entre contrat conventionnel et contrat HMO. On peut conclure qu'un tiers de cette différence est dû à l'innovation liée à la HMO. Ce même tiers sert comme facteur de répartition pour déterminer la part de diminution de dépenses de $9 \%$ qui résulte d'une fréquence réduite d'initialisations.

En ce qui concerne la différence après initialisation de 53\%, la répartition entre innovation et sélection se base sur les tableaux 3 et 5 , qui mettent en évidence une diminution des dépenses de santé de $37 \%$ par rapport à celles des assurés avec un contrat conventionnel, et cela après insertion de la santé latente dans l'équation. Comme cette variable est censée neutraliser les effets de la sélection, cette diminution de $37 \%$ est créditée à l'innovation, en particulier aux changements d'incitation des médecins adhérant à une HMO. Le restant des 53\%, c'est-à-dire la différence de $16 \%$, est attribuée aux effets de la sélection.

En faisant l'addition, on arrive à la répartition suivante entre les effets « innovation» et «sélection». La différence brute des dépenses de santé de $62 \%$ en faveur de la HMO se répartit en $40 \%$ dus à l'innovation et $22 \%$ dus à la sélection 
des risques dans le cas de la HMO. Pour l'IPA, la diminution brute est de $34 \%$ et elle est entièrement attribuable à une réduction des dépenses de santé après initialisation du traitement. Selon les tableaux 5 et 6, l'effet IPA n'atteint $10 \%$ que si la santé latente figure parmi les variables explicatives. La répartition entre les deux effets s'avère donc moins favorable, avec une diminution des dépenses attribuables à l'innovation de $10 \%$ et à la sélection des risques de $24 \%$. Enfin, le calcul est très similaire pour la PPO. La différence brute de $39 \%$ peut être attribuée pour $21 \%$ à l'innovation et pour $18 \%$ à la sélection.

En somme, ces estimations suggèrent que la réduction très impressionnante des dépenses de santé obtenue par les contrats de type HMO d'un assureurmaladie suisse est attribuable pour deux tiers à des innovations telles que les modifications des incitations des médecins et la pratique en groupe combinée avec la surveillance mutuelle. La PPO, qui est constituée par une liste de praticiens excluant les médecins les plus coûteux, suit en deuxième rang. Ici les réductions de dépenses proviennent de l'innovation et de la sélection à peu près à parts égales. Finalement, la variante IPA, qui réunit des médecins indépendants agissant comme surveillant de la filière des traitements, semble profiter d'importants effets de sélection alors que l'innovation ne serait responsable que d'un seul tiers de la réduction observée des dépenses.

\section{RÉSUMÉ ET CONCLUSION}

Cette conférence a eu comme point de départ le concept de la sélection biologique. Elle a cherché à clarifier la relation existant entre les concepts biologique et économique de sélection. En effet, la théorie économique décrit les conditions qui règlent la survie d'une entreprise sous la pression de la concurrence. Un marché concurrentiel constitue donc un mécanisme de sélection en ce sens que, du côté la demande il ne retient comme consommateurs effectifs que les individus munis d'une disponibilité à payer égale ou supérieure au prix de marché et, du côté de l'offre, il ne retient comme vendeurs effectifs que les producteurs atteignant un coût de production unitaire inférieur ou égal au prix de marché. C'est parce que l'équilibre d'un marché concurrentiel est efficient au sens de Pareto que ce processus de sélection s'avère être une condition cruciale à l'obtention de l'efficience. Quoique peut-être plus surprenant, le même constat s'impose au sujet de la sélection des risques dans le marché d'assurance, bien que la sélection des risques soit entièrement rejetée par l'assurance maladie sociale.

En partant du principe que la sélection des risques est une chose à éviter, au moins dans le cadre de la régulation des primes actuelle, on peut s'interroger sur le rôle joué par les nouvelles variantes contractuelles du type «managed care » qui ont été lancées ces dernières années. Une variante, offerte par un assureur-maladie social en Suisse et correspondant à la formule de la «Health Maintenance Organization (HMO)» aux Etats-Unis, réalise une réduction des dépenses de santé de $62 \%$, par rapport au contrat conventionnel. Est-ce que cette diminution est la conséquence d'une concentration des risques faibles dans ce type de contrat, ou est-elle la conséquence d'innovations, comme la modification des incitations des médecins à réduire les actes médicaux lorsqu'ils sont payés au forfait plutôt qu'à l'acte? 
Une étude récente utilise des observations historiques pour construire un indicateur de l'état de santé latente permettant de rendre compte des différences entre risques élevés et risques faibles. Après estimation de cette différence, deuxtiers de la réduction de $62 \%$ des dépenses de santé peuvent être attribués à l'effet innovation du contrat HMO. Les deux autres alternatives de «managed care» permettent une réduction des dépenses de santé nettement inférieure et la part attribuable à l'innovation ne représente plus que la moitié, voire le tiers de cette réduction.

De tels résultats invitent à la réflexion. En premier lieu, la péréquation des risques semble produire des effets secondaires non désirés, car elle ne prend pas en compte le fait que toute innovation tend à attirer les assurés non captifs, dont la majorité sont des jeunes. Or, une augmentation du nombre de jeunes donne lieu à une augmentation des paiements au fonds de péréquation. Ainsi, la péréquation a pour effet de contrer l'innovation et qui plus est, elle affecte les différents types d'innovation de la même façon, bien que nos analyses suggèrent des différences importantes dans les contributions respectives des effets d'innovation et de sélection de risques à la réduction des dépenses de santé.

En deuxième lieu, la mise en place d'une assurance maladie unique, gérée par un monopole public, comme le préconisent certains courants politiques en Suisse, aurait pour conséquence de neutraliser l'effet de la sélection des risques par manque d'innovation. Tout au moins, une assurance maladie unique aurait de la peine à développer de nouvelles variantes contractuelles, si elles sont basées sur une sélection des risques à l'intérieur de l'organisme. Dès lors, la question est de savoir si les innovations, toujours imparfaites, sont suffisamment importantes pour justifier le refus d'un monopole public.

Ces grandes questions restent sans réponse dans cette conférence. Mais les enjeux qu'elles sous-tendent apparaissent beaucoup plus clairement grâce aux progrès réalisés par l'économétrie, qui permettent de rendre compte des processus de sélection. A présent, les problèmes causés par le manque de représentativité des échantillons de données peuvent souvent être résolus. Malheureusement, Luigi Solari ne vit plus pour voir ces développements, mais il aurait sans doute été fasciné par les potentialités de ces nouvelles méthodes.

Université de Zurich

pzweifel@unizh.ch

\section{RÉFÉRENCES BIBLIOGRAPHIQUES}

Alchian A. (1950), «Uncertainty, evolution, and economic theory », in: Journal of Political Economy, 58.

Berg, S.V. \& Tschirhart, J. (1988), Natural Monopoly Regulation: Principles and Practice, Cambridge: Cambridge University Press.

Darwin C. (1871), The Descent of Man and Selection in Relation to Sex, London: J. Murray.

Dawkins R. (1976), The Selfish Gene, New York: Oxford University Press.

Dubin J.A. et McFadden D.L. (1984), «An econometric analysis of residential electric appliance holdings and consumption », in: Econometrica, 52, 345-62. 
Frank R.H. (2000), Microeconomics and Behavior, $4^{\mathrm{e} m e}$ édition., Boston, McGraw-Hill.

Hirshleifer J. \& Riley J.G. (1979), «The analytics of uncertainty and information», in: Journal of Economic Literature, XVII, 1375-1421.

Lee L.F. (1983), «Generalized econometric models with selectivity», in: Econometrica, 51, 507-512.

Lehmann Hj. (2002), Managed Care: Kosten senken mit alternativen Krankenversicherungsmodellen? (Managed Care: Baisser les coûts par de nouveaux modèles d'assurance maladie?), Thèse de doctorat, Université de Zurich.

Mas-Colell A., Whinston M.D. \& Green J.R. (1995). Microeconomic Theory, New York: Oxford University Press.

Van de Ven W.P.P.M. \& Ellis R.P. (2000), «Risk adjustment in competitive health plan markets», in: Culyer, A.J. et Newhouse, J.P. (éds), Handbook of Health Economics, Vol. Ia, Amsterdam: Elsevier, 755-845. 